\title{
AOR
}

Selected Papers of \#AolR2021:

The 22nd Annual Conference of the Association of Internet Researchers Virtual Event / 13-16 Oct 2021

\section{SEARCHING FOR THE LOST: EXAMINING THE ONLINE PRESENCE OF VICTIMS OF POLICE KILLINGS}

\author{
Jennifer Pierre \\ YouTube \\ Morgan Currie \\ University of Edinburgh \\ Britt Paris \\ Rutgers University \\ Irene Pasquetto \\ University of Michigan

\section{Introduction}

Contrary to the idea that social media is solely or primarily used for recreation, American adults are increasingly using social media for a variety of personal informational and expressive tasks, including finding and sharing health information, advice on parenting and dating, and even information on stress levels (Perrin, 2015). Many scholars point toward a deeper and more comprehensive study of social media in general as a worthy goal of continued future research in the information fields (boyd, 2015; Brabham, 2015; Domingo, 2015). This becomes increasingly important when investigating specialized contexts of social media use among marginalized populations, where less is known and values and motivations around social media use may shift (Pierre, 2019).

One specific area of non-recreational social media use, mourning or bereavement, is a growing but still limited area of inquiry. Aiming to contribute to this body of work, this study investigates the basic online presence of individuals who have been killed by law enforcement through an analysis of their social media accounts or accounts of those connected to them. Further, we explore how social media data might inform the incidents surrounding these victims' deaths. The motivating inquiry behind our work is whether the presence of and content within social media accounts can contribute to a better understanding of the lives of victims of police killings, with a specific goal of using

Suggested Citation (APA): Pierre, J., Currie, M., Paris, B., Pasquetto, I. (2021, October). Searching For the Lost: Examining the Online Presence of Victims of Police Killings. Paper presented at AolR 2021: The 22nd Annual Conference of the Association of Internet Researchers. Virtual Event: AoIR. Retrieved from http://spir.aoir.org. 
this information to better understand how social media platforms and data can be used to support affected communities. We specifically acknowledge the role that this social media content can play in the lives of BIPOC and specifically Black communities, as a counter-data archive to help more thoroughly reflect their lived realities as communities severely disproportionately affected by police violence (The Guardian 2016, Mapping Police Violence. 2019).

\section{Background}

Our paper describes one portion of a larger research venture, the Police Officer Involved Homicide (POIH) Project. As part of this work, our project team assessed and analyzed available federal and local databases that collected information on POIH. After finding significant gaps in consistency of and access to collected information across the databases, we invited community members to work on decoding and understanding the datasets with us, as a way of ideally better accommodating data use for community desires and needs.

The working group of particular interest in this paper is the social media mining group. Participants on this team manually searched through Facebook, Twitter, YouTube, and other platforms to find any information outside of traditional news sites regarding victims of police killings. The goal was to find and uncover the narratives surrounding victims from a more personal and nuanced perspective, through the social media accounts of family and friends, the reactions and comments of average citizens or witnesses, or even information provided from the victims prior to their passing.

This paper describes the process of discovering and naming the three emergent categories of online presence we observed from this process, explains the specificities of the categories themselves, and discusses how these findings might aid in better understanding the varied contexts involved in police killings. As scholars like Boyd \& Crawford (2012) ascertain, the need for critical assessment of big data usage is of increasing importance as it plays a larger role in research and policymaking, including the regulations regarding $\mathrm{POIH}$. We ultimately hope that this piece of our project, as well as the project as a whole, will positively contribute to the ability of affected community members to work with the available data on police killings in ways that empower and educate them, and serve their needs if desired.

\section{Method}

The set of methods guiding the social media mining element of our POIH database project involved primarily manual social media mining, followed by a qualitative analysis of found sites, accounts, and posts to gain a clearer comprehension of each victim's presence across platforms.

\section{Findings and Discussion}

Our reflections on the social media mining portion of our $\mathrm{POIH}$ database analysis project fall into two major categories: emergent themes gathered from the social media content collected and reviewed for each victim, and overall takeaways on the inclusion 
of social media analysis as part of a suite of methods for collective use in engagement with and analysis of POIH data. In general, the patterns we observed in the types of social media content found for each of the deceased fell into three categories:

persistence of the deceased's activity across social media, sensationalized commentary on videos and blog postings, and memorials on Facebook, Tumblr, and Twitter.

Persistence of the deceased's activity: Information offered in this category about the victim typically did not directly relate to the details surrounding their death, but rather revealed the normal day-to-day activities and conversations in which they may have engaged. It served as a both a source of online preservation for the individual, as well as a contribution to a broader narrative.

Sensationalized commentary: Examples of sensationalized commentary, or comments consisting of provocative language often portraying an extremist view, were observed in general in the comment sections of videos of POIH footage. The comments offer very little substantive information to the context of the taped $\mathrm{POIH}$, and instead attempt to detract from the potential importance and significance of the videos as evidence of police brutality by focusing on speculation and opinion based commentary. They signal a greater need for deeper comprehension of qualitative and quantitative data on these victims.

Memorials: The final type of social media trace found on deceased POIH individuals were memorials found primarily on Facebook, Twitter, and Tumblr. They provide a gathering space for the contribution of information from individuals close to the source, the deceased's family and friends, and combine the basic details of the deceased's death with the potential for honest and knowledgeable reflections on the controversial issues surrounding the death.

Each of the types of content described and analyzed plays a particular role in adding context, nuance, and layers of information and evidence to the lives of police victims. We use this analysis to advocate for considering this information as part of an assemblage of relevant information that exists in addition to the more widely or primarily considered police and federal reports. This may substantially enhance the ability for more nuanced understanding of police killing incidents, which may in turn affect the ongoing discussions regarding ways to improve the standards and regulations they work within. These data add necessary complexity to way these incidents are presented and consumed as data points, and can be an important tool for empowerment, understanding, and healing for communities most affected, in particular Black and other POC communities.

\section{References}

boyd, D. (2015). Social Media: A Phenomenon to be Analyzed. Social Media+ Society, $1(1)$.

Boyd, D., \& Crawford, K. (2012). Critical questions for big data: Provocations for a cultural, technological, and scholarly phenomenon. Information, communication \& society, 15 (5), 662-679. 
Brabham, D. C. (2015). Studying Normal, Everyday. Social Media+ Society , 1(1), Social Media. 2056305115580484.

Domingo, D. (2015). Follow Them, Closely. Social Media+ Society, 1( 1), 2056305115578134.

Mapping Police Violence, 2019

Perrin, A. (2015). Social Media Usage: 2005-2015.

Pierre, J. (2019). Building a Digital Family: Examining Social Media and Social Support in the Development of Youth "At-Risk" (Doctoral dissertation, UCLA).

The Counted, The Guardian, 2016 